\title{
RESOURCE SUPPLY AN ACCESSIBILITY OF PUBLIC TRANSPORTATION FACILITIES
}

\author{
Eshetu Mathewos Juta \\ Assistant Professor, Civil Engineering Department, Wolaita Sodo University, Wolaita Sodo, \\ Ethiopia \\ Email Id: eshetum90@gmail.com
}

\begin{abstract}
The term "urban mass transit" generally refers to scheduled intra-city service on a fixed route in shared vehicles. Public transportation is an important contributing factor to urban sustainability. Effective transportation networks that incorporate public transit livable by easing commute and transportation needs and increasing accessibility. To assess public transportation accessibility in metropolitan networks, two indices are used: the supply level of urban public transportation facilities resource and the public transportation-private automobile traveling time ratio. As the research in the Wolaita sodo town region and the assessment system, an evaluation technique for urban public transportation facility resource supply is developed based on accessibility. Accessibility is a representative indicator for evaluating the supply of bus system. Traditional studies have evaluated the accessibility from different aspects. Considering the interaction among land use, bus timetable arrangement and individual factors, a more holistic accessibility measurement is proposed to combine static and dynamic characteristics from multisource traffic data. The objective is to highlight the main lessons learned and identify knowledge gaps to guide the design and evaluation of future transport investments. Moreover, studies looking at ways to improve the operational efficiency of systems and those seeking to promote behavioral changes in transport users offer great potential to generate learning that is useful for the public and private actors involved.
\end{abstract}

Keyword: Urban Planning, transportation traffic, environment impact, design

\section{Introduction.}

Accessibility has been used in a variety of sectors, including urban and land use planning, transportation management, and public facility site studies. The classical location theory was the first investigation on accessibility. The impacts of greenhouse gasses, for example, environmental pollution, respiratory diseases, and global warming, are well documented. Many factors contribute to increased levels of these harmful gasses, including cutting down trees, the use of fossil fuels, and emission from industries and vehicles. One factor that is consistently ignored, yet contributes more to greenhouse emissions, is personal transportation, driving personal cars to be precise. Those living in highly populated cities feel the impacts of emissions by vehicles. And with technology traveling at lung-bursting speed coupled with rapidly expanding middle class, car ownership is projected to skyrocket. This means the universe will be polluted more than ever. The only way to reverse this trend is the adoption of public transportation. 
From the standpoint of urban area and land use, accessibility can be used to calculate space among different areas and to analyze the convenience of reaching other places from the current area; from the standpoint of transportation system, accessibility can be used to calculate space among different areas and to analyze the convenience of reaching other places from the current area; from the standpoint of transportation system, accessibility can be used to calculate space among different areas and to analyze the convenience of reaching.

Cities are locations having a high level of accumulation and concentration of economic activities. They are complex spatial structures supported by infrastructures, including transport systems. The larger a region, the greater its complexity and the potential for disruptions, particularly when this complexity is not effectively managed. Urban productivity is highly dependent on the efficiency of its transport system to move labor, consumers, and freight between multiple origins and destinations. Additionally, transport terminals such as ports, airports, and railyards are located within urban areas, help anchor a city within a regional and global mobility system. Still, they are also contributing to a specific array of challenges

\subsection{Traffic congestion and parking difficulties}

Congestion is one of the most prevalent transport challenges in large urban agglomerations. Although congestion can occur in all towns (Ethiopia). Congestion is particularly linked with motorization and the diffusion of the automobile, which has increased the demand for transport infrastructures. However, the supply of infrastructures has often not been able to keep up with mobility growth. Since vehicles spend the majority of the time parked, motorization has expanded the demand for parking space, which has created footprint problems, particularly in central areas where the footprint of parked vehicles is significant. Congestion and parking are also interrelated since street parking consumes transport capacity, removing one or two lanes for circulation along urban roads. Further, looking for a parking space called cruising creates additional delays and impairs local circulation. In central areas of large cities, cruising may account for more than $10 \%$ of the local circulation, as drivers can spend up to 20 minutes looking for a parking spot.

\subsection{Longer commuting}

On par with congestion, people are spending an increasing amount of time commuting between their residence and workplace. An important factor behind this trend is related to residential affordability as housing located further away from central areas (where most of the employment remains) is more affordable. Therefore, commuters are exchanging commuting time for housing affordability. However, long commuting is linked with several social problems, such as isolation less time spent with family or friends, as well as poorer health (obesity). Time spent during commuting is at the expense of other economic and social activities. However, information technologies have allowed commuters to perform a variety of tasks while traveling. 


\subsection{Public transport inadequacy}

Many public transit systems, or parts of them, are either over or underused since the demand for public transit is subject to periods of peaks and troughs. During peak hours, crowdedness creates discomfort for users as the system copes with a temporary surge in demand. This creates the challenge of the provision of an adequate level of transit infrastructures and service levels. Planning for peak capacity leaves the system highly under-used during off-peak hours, while planning for an average capacity will lead to congestion during peak hours.

Low ridership makes many services financially unsustainable, particularly in suburban areas. Despite significant subsidies and cross-financing (e.g. tolls), almost every public transit system cannot generate sufficient income to cover its operating and capital costs. While in the past, deficits were deemed acceptable because of the essential service public transit was providing for urban mobility, its financial burden is increasingly controversial.

\subsection{Difficulties for non-motorized transport}

These difficulties are either the outcome of intense traffic, where the mobility of pedestrians, bicycles, and other non-motorized vehicles is impaired, but also because of a blatant lack of consideration for pedestrians and bicycles in the physical design of infrastructures and facilities. On the opposite side, the setting of bicycle paths takes capacity away from roadways as well as parking space. A negative outcome would be to allocate more space for non-motorized transport than the actual mobility demand, which would exacerbate congestion.

\subsection{Loss of public space}

Most roads are publicly owned and free of access. Increased traffic has adverse impacts on public activities, which once crowded the streets such as markets, agoras, parades and processions, games, and community interactions. These have gradually disappeared to be replaced by automobiles. In many cases, these activities have shifted to shopping malls, while in other cases, they have been abandoned altogether. Traffic flows influence the life and interactions of residents and their usage of street space. More traffic impedes social interactions and street activities. People tend to walk and cycle less when traffic is high.

\subsection{High infrastructure maintenance costs}

Cities facing the aging of their transport infrastructure have to assume growing maintenance costs as well as pressures to upgrade to more modern infrastructure. In addition to the involved costs, maintenance and repair activities create circulation disruptions. Delayed maintenance is rather common since it conveys the benefit of keeping current costs low, but at the expense of higher future costs and, on some occasions, the risk of infrastructure failure. The more extensive the road and highway network, the higher the maintenance cost and its financial burden. The same applies to public transit infrastructure that requires a system-wide maintenance strategy. 


\subsection{Environmental impacts and energy consumption}

Pollution, including noise generated by circulation, has become an impediment to the quality of life and even the health of urban populations. Further, energy consumption by urban transportation has dramatically increased, and so the dependency on petroleum. These considerations are increasingly linked with peak mobility expectations where high energy prices incite a shift towards more efficient and sustainable forms of urban transportation, namely public transit. There are pressures to "decarbonize" urban transport systems, particularly with the diffusion of alternative energy sources such as electric vehicles.

\section{Urban Public Transport Resource Supply}

Public transportation contributes to a healthier environment by improving air quality and reducing oil consumption, and through better land-use policies. It also helps to expand business development and work opportunities. And, it is critical for emergency situations requiring safe and efficient evacuation. Why is public transport important essay? One major benefit of using public transport is that it reduces the number of vehicles running on roads and gradually slows down pollution. When use of public transport will increase, there will be fewer private vehicles on the road. Walking range and covered stops are set by passengers' maximum tolerable walking duration. The accessibility of demand points may then be reached by calculating the overall accessibility of stations within walking distance of all demand locations while considering station type, number of routes, weight, and walking walk-to-public transit-stop duration into consideration. Finally, the public transport-private car travelling time ratio is calculated using the network map and the public transport-private car travelling time to reflect the relative service competitiveness of public transportation and private cars and to allow evaluation of the public transportation accessibility of major areas. Figure 1 depicts the technical path.

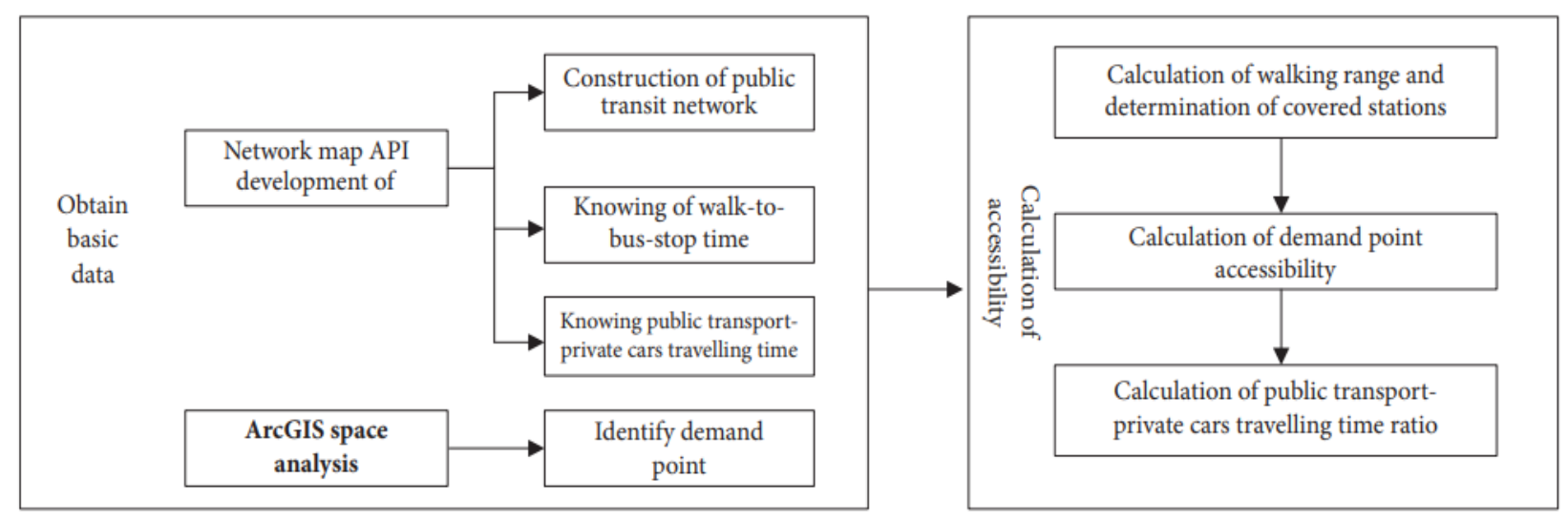

Figure 1: Technology roadmap

\subsection{Network of Public Transportation.}

Because the urban public transportation network is always evolving and being altered, it is difficult to obtain real-time and up-to-date basic network data. Online maps such as Baidu, 
Google, and Amap give a wealth of geographic information, and their data updates quickly and comprehensively. The study uses Baidu map as an example to create an urban public transit network capture system by utilizing the Baidu development interface and secondary development of the ArcGIS Engine, which has successfully captured Ethiopia public transportation and transit network data, which primarily includes public transportation station data (station name as well as longitude and latitude) and route data. This method is applicable to all domestic region are covered by network maps in order to gather accurate and complete public transportation network data.

\section{Literature review}

Akinyemi (2000) that sustainable mobility requires, among other things, acceptable levels of environment impacts and costs of development and operation of transportation system etc. three main inferences can be made from these ideas. The first inference is that sustainable mobility is significantly transportation supply dependent. The better and more sustainable the supply characteristics of a transportation system, higher will be the level of mobility of peoples. The second inference is that current levels of people mobility in many developing cities can be said to be low and unsustainable largely because of inadequate transportation supply characteristics. The inadequacy of transportation supply in many countries manifests mainly through inadequate public transportation services, low productivity and level of ride ability of facilities and high level of transportation related environmental impacts. For example, in many cities, it is difficult to move around by any mode of transportation wit out physically and/or mentally exhausted in the process.

Whether or not cities, which are hubs connecting markets such as those on labour, investment, education, commerce, recreation and health care, etc., can prosper depends, among others, on the accessibility of these markets. The accessibility, in turn, is influenced by the availability of an efficient and effective transport system. What is an effective and efficient urban transport system? It is one that can satisfy the numerous and diverse requirements of the metropolitan mobility, including minimizing travelling time between various locations, while at the same time internalises externalities to positively affect the well-being and the quality of life of the citizens of that area. Among the most apparent externalities are traffic congestion, traffic accidents and environmental pollution including climate change. The larger the metropolitan area, the greater is its complexity and the potential for transport externalities and therefore for transport disruptions. Introducing and sustaining an effective and efficient transport system is therefore not an easy but possible task. This requires that the appropriate authorities transform the available land and financial resources, both limited capital inputs, into relevant transport infrastructure and networks to provide, what is considered, the right combination between the various motorized individual or collective/public and non-motorized transport modes, as well as easy transfers between them to meet the mobility demand.

The major modes of public transportation in the town are zeman bus and selam bus. These providers are hardly able to cope with public's demand for transportation. Thus, residents of wolaita sodo have to face great inconveniences, as well as additional costs to the daily trips to 
their destinations. This study attempts to identify the major factors that contribute to the high demand for public transport system in the city. This study uses descriptive research method. Hence, it mainly relies on secondary data, whereas primary data used as supplementary. The increasing population size and physical expansion of Wolaita sodo largely contributes to the demand for public transport. Social and economic purpose trips also contribute to the demand for public transport in the city. However, the existing public transportation system could not satisfy the demand. Moreover, the insufficient finance for investment by the government and the limited participation of the private sector in the service has also contributed for deficiency of transport supply in the city.

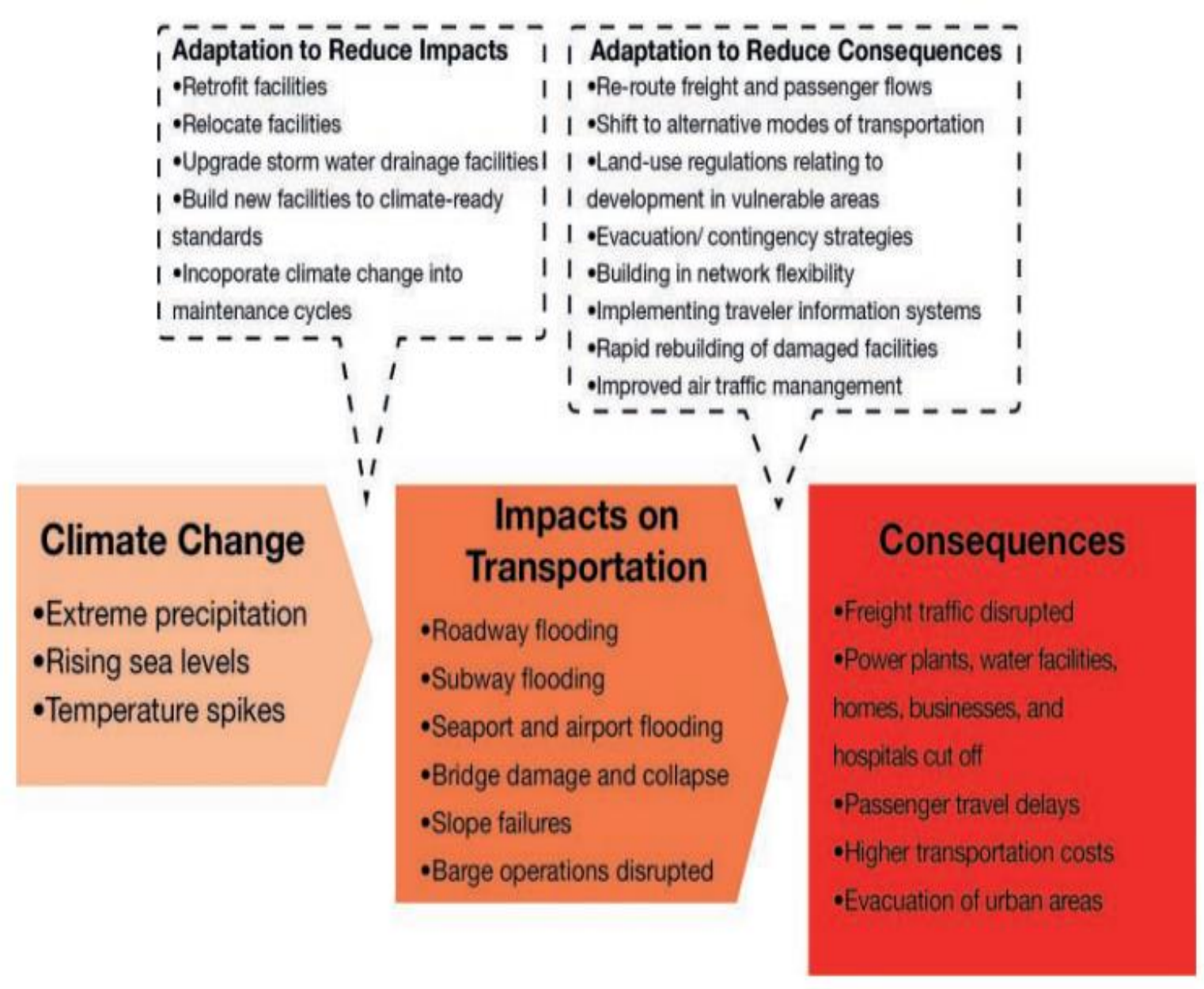

Figure 2: Public Transportation Facilities Resource Supply Level

\subsection{Public Transportation Accessibility}

Transportation is now the largest source of carbon emissions in the Ethiopia. In many towns, the personal automobile is the single greatest polluter because emissions from millions of vehicles on the road add up. To reduce greenhouse gas emissions, individuals can use cleaner modes of transportation to get around, from public transit to biking and walking. Transportation (29 percent of 2019 greenhouse gas emissions) - The transportation sector generates the largest share of greenhouse gas emissions. Greenhouse gas emissions from transportation primarily come from burning fossil fuel for our cars, trucks, ships, trains, and planes. 


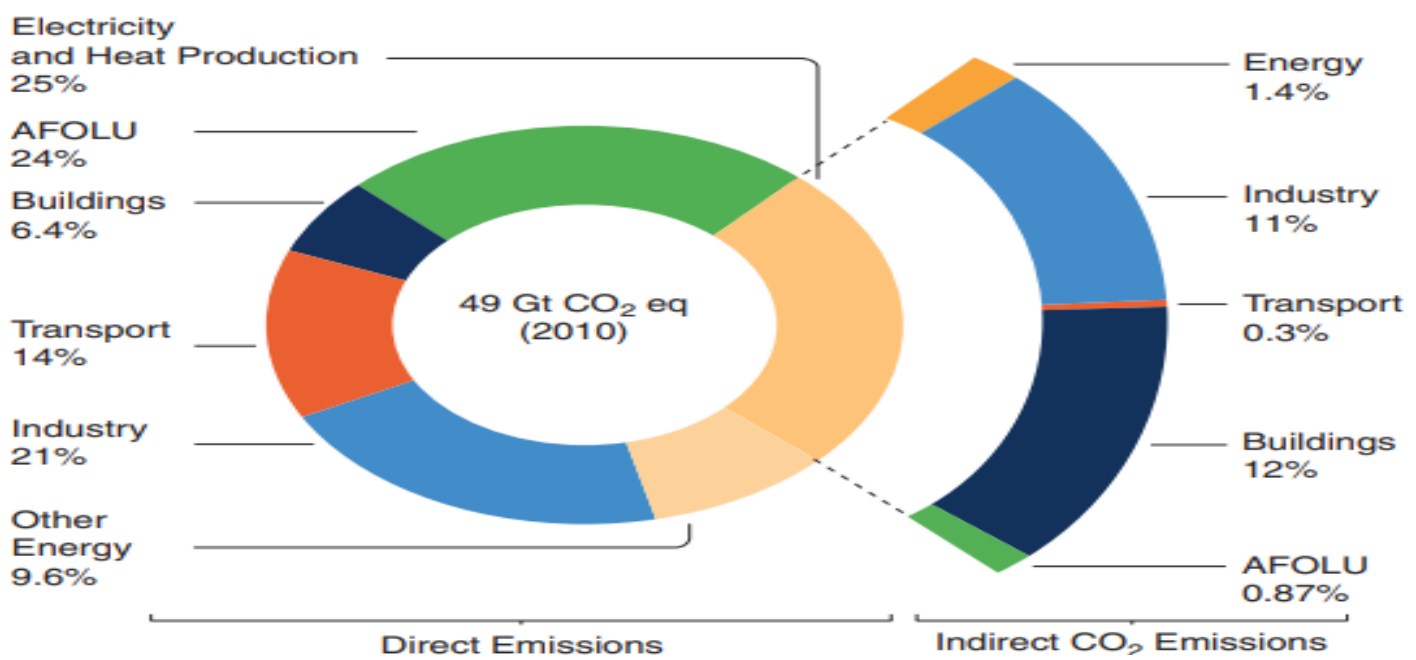

Figure 2: Urban transport's greenhouse gas emissions among economic sectors

\subsection{Public transportation policy based on population density.}

To some extent, the distribution of public transportation resources affects regional development opportunities; the public transport accessibility evaluation method proposed in this paper is combined with population density and structure distribution, which can provide decision-making support for balanced public transportation development. Figure illustrates that Ethiopia population is evenly dispersed, with significant population density in both the center region and outlying streets Figure illustrates that public transportation resources are not fairly dispersed in outlying regions; public transportation accessibility in certain densely populated areas is limited, which requires additional attention. Of course, in addition to population density, greater attention should be made to differences in population structure and public transportation demand.

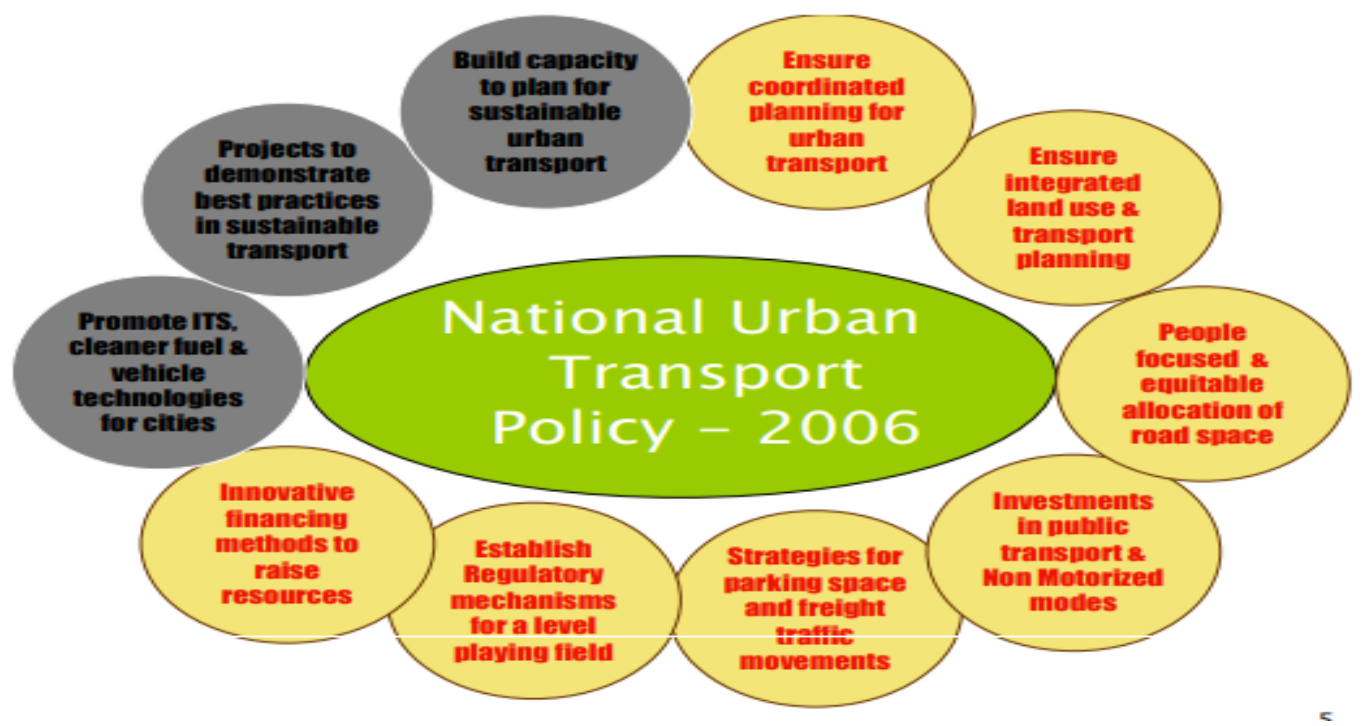

Figure 3: Public transportation policy based on population density. 


\section{Urban Planning, Transport Engineering, and Design Solutions}

New policies, regulations, and codes that address transportation system vulnerabilities to mainstream adaptation strategies in the planning, design, and execution of transportation projects. With long-term environmental changes likely to expose urban transportation infrastructure to intense and frequent floods, strong winds, and rising temperatures, cities should consider mitigating risks by incorporating high-probability scenarios into the design of transportation systems and associated citywide interdependent systems.

These principles and processes apply to both new capital-intensive investments and routine retrofits of rail systems, highways, bridges, automobiles, and other interconnected urban systems with 50-100-year life expectancies. The majority of the literature on urban transportation adaptation focuses on supply-side planning, engineering, and design solutions. However, land-use planning provides a considerable opportunity for adaption options. Land-use planning can help with adaptation and mitigation in some circumstances. The mutually interdependent links between land use and transportation networks, as well as the accompanying GHG reductions, are discussed. The distribution of population density and mix of activities connected with urban land use are determined by land use, zoning, and construction laws, as well as other urban development policies. Travel demand is shaped by non-transport urban development initiatives. However, there is little examination of the adaptive potential of such land- and real-estate-derived policy instruments in the extant research and policy literature. Similarly, the adaptability of emerging flexible and information technology-enabled flood response systems that can respond to real-time changes in travel demand or structure elevation should be investigated, as should low-cost and low-technology solutions, especially for resource-constrained areas.

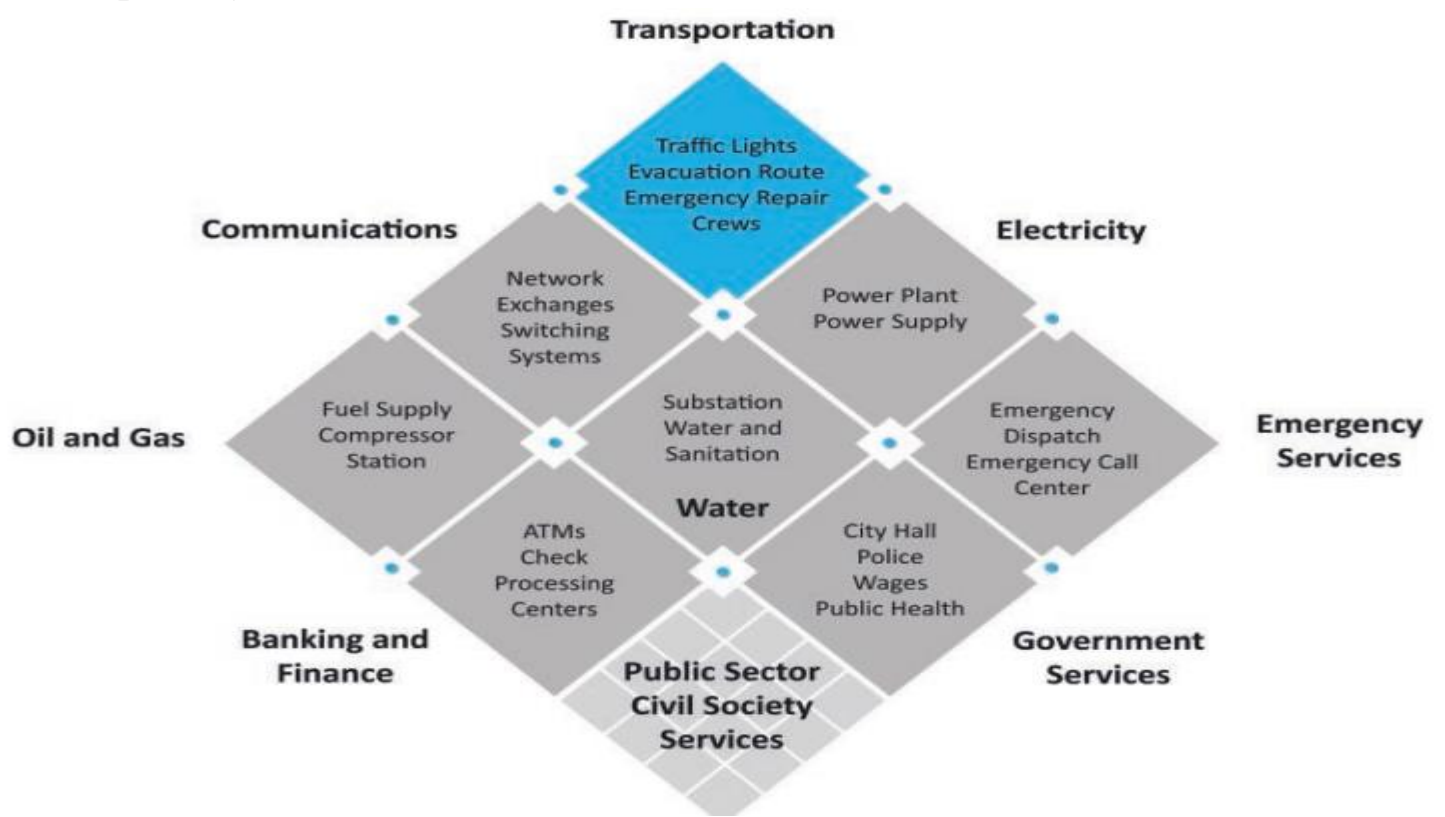

Figure 4: Urban transport's interconnectivity with urban systems.

In many towns in Ethiopia, urban transportation adaptation necessitates a thorough re-evaluation of design practices and procedures. Climate change risk assessment, as well as the identification and mitigation of threats, are among them. Choosing the right combination of 
policies, programs, and initiatives, and tactical reactions that are measured in the short and long term fresh research should be included, and maintenance and operations should be re-calibrated asset management and processes

\section{Conclusions.}

This study provides an urban public transportation resource supply evaluation approach based on accessibility, which takes into account passenger walking time to stations as well as other parameters such as station property, class, and geographic distribution features. Equity is a key component of a long-term urban transportation system. The method proposed in this paper essentially reflects the distribution of urban public transportation facilities resource supply, evaluates the equity of urban public transportation facilities resource distribution based on the accessibility index, calculates the relative service competitiveness of public transportation and private cars based on the index of public transport-private cars traveling time ratio, and provides conditions for the public transport accessibility evaluation of. Using Shenzhen as an example, the proposed method is demonstrated. The result demonstrates that the method accurately reflects the spatial distribution characteristics of Shenzhen public transportation facilities and can be used to develop new methods.

Therefore, public transit systems are challenged to remain relevant to urban mobility as well as to increase its market share. The increasing volatility in petroleum prices since 2006 provides uncertainties in the costs of transit fleet ownership and operations and how effective it is to convert transit fleets to alternative energy sources. A younger generation with a preference in living in higher density areas perceives the automobile as a less attractive proposition than the prior generations. Electronic fare systems are also making the utilization of public transit more convenient. A recent trend concerns the usage of incentives, such as point systems (e.g. air miles with purchase of a monthly pass), to promote public transit and influence consumer behavior. Yet, evidence underlines that the inflation-adjusted cost of using public transit is increasing, implying that the cost advantage of public transit over the automobile is not changing in a significant manner.

\section{References}

[1] Akinyemi, "sustainable mobility in urban area" science direct journal, 3: 2 Pp 30-45, 2006

[2] Tatenda mbara," Mobility Challenges for the 21st Century" journal of Urban Mobility and Poverty Reduction ISSN: 2279-1644, Sep-2010.

[3] Mega Kumar, Seema Singh, Akshima T Ghante, Sarbojit pal, Sangeetha Ann Wilson, "Informal public transport mode in India: a case study of five city region". IATSS research, 2016.

[4] A publication on "Sustainable Urban Mobility andPublic Transport In UNECE Capitals" Transport trends and economic series; 2015. 
[5]. Avishai (Avi) Ceder"Optimal Multi-Vehicle Type Transit Timetabling and Vehicle Scheduling” Procedia Social and Behavioural Sciences, 20, 2011.

[6]. Spada, M.; Bierlaire, M. and Liebling, T. M. (2005). "Decision-aiding methodology for the school bus routing and scheduling problem". Transportation Science, vol. 39, No. 4 (November), pp. 477-490.

[7]. Braca, J.; Bramel, J.; Posner, B. and Simchi-Levi, D. (2017). "A computerized approach to the school bus routing problem”. IIE Transactions, vol. 29, No. 8, pp. 693-702. 